\title{
Influence d'une injection intraveineuse de L-tryptophane et/ou de L-arginine sur le taux plasmatique de l'hormone de croissance $(\mathrm{GH})$ chez l'agneau
}

\author{
R Guilhermet ${ }^{1}$, J Charrier ${ }^{2}, \mathrm{R}$ Toullec ${ }^{1}$ \\ 1 INRA-ENSA, laboratoire du jeune ruminant \\ 65, rue de Saint-Brieuc, 35042 Rennes Cedex; \\ 2 Unité de différenciation cellulaire et croissance, \\ 9, place Viala, 34060 Montpellier Cedex, France
}

\begin{abstract}
Summary - Effect of an intravenous injection of L-tryptophan and (or) L-arginine on the plasma level of growth hormone (GH) in the lamb. Ruminant lambs were given intravenous infusion of amino acids. L-Arginine increased the plasma level of the growth hormone. L-Tryptophan used at a low level produced only a small GH increase. No synergic effect of the 2 amino-acids was observed.
\end{abstract}

L'effet stimulateur de l'arginine sur la sécrétion de GH est connu depuis longtemps; il s'exerce aussi bien chez l'homme (Knopf et al, 1965) que chez le mouton (Davis, 1972). Le tryptophane est aussi susceptible de favoriser la sécrétion de $G H$, via une stimulation du système sérotoninergique, chez le monogastrique, mais on ne dispose pas de données chez le jeune ruminant en croissance; par ailleurs, l'effet de ces acides aminés administrés en association n'est pas connu. Le but de cet essai est d'étudier l'influence de ces acides aminés sur la sécrétion de $\mathrm{GH}$ chez l'agneau.

Matériel et méthodes - Quatre agneaux mâles Lacaune de $15 \mathrm{~kg}$ de poids vif reçoivent $500 \mathrm{~g}$ de lait de remplacement $(105 \mathrm{~g}$ de matière sèche) par jour, ainsi qu'un aliment concentré et du foin, ad libitum. Ils sont munis d'un cathéter placé dans une veine jugulaire. Ils sont utilisés selon un dispositif en carré latin $4 \times 4$ comportant l'injection intraveineuse de $1 \mathrm{ml} / \mathrm{kg}$ de poids vif de solutions à $1 \%$ de $L$-tryptophane $(T), 10 \%$ de $\mathrm{L}$-arginine (A), $1 \%$ de L-tryptophane $+10 \%$ de Larginine $(T+A)$ ou $0,9 \%$ de chlorure de sodium (C). Les solutions sont injectées en $3 \mathrm{~min}$, à $10 \mathrm{~h}$ du matin. Cinq millilitres de sang sont prélevés 60 et $10 \mathrm{~min}$ avant l'injection et $5,12,20,30,45,60,90,120,180$ et $240 \mathrm{~min}$ après. Les journées de mesure sont séparées d'au moins $72 \mathrm{~h}$. La GH est dosée par radio-immunologie, par une méthode à double anticorps, I'oGH-NIH-S-5 étant utilisée comme hormone froide et traceur radio-actif.

Résultats et discussion - Le taux plasmatique de $\mathrm{GH}$ augmente après l'injection des solutions $\mathrm{A}$ et $\mathrm{T}+\mathrm{A}$ (maximum à $12 \mathrm{~min}$ ) et revient au niveau de base au bout de 90 ou $120 \mathrm{~min}$ (fig 1). La solution $T$ produit une augmentation nettement moins forte que les solutions contenant l'arginine. La hauteur moyenne des pics est plus éle- 


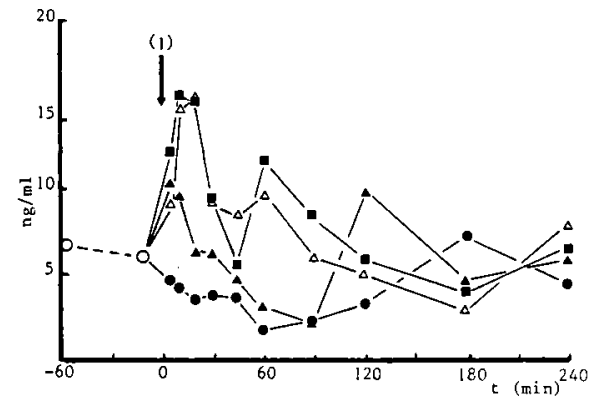

Fig 1. Évolution des concentrations plasmatiques de $\mathrm{GH}$ chez l'agneau recevant par voie intraveineuse (I) un bolus de solution saline $(\bullet)$, de L-tryptophane $(\mathbf{\Delta})$, de L-arginine $(\boldsymbol{\square})$ et de mélange L-tryptophane + L-arginine $(\Delta)$.

vée $(P \leq 0,05)$ avec les solutions $A$ et $\mathrm{T}+\mathrm{A}$ quavec les solutions $\mathrm{T}$ et $\mathrm{C}$ (respectivement $10,3 \pm 1,1,10,9 \pm 1,4$, $7,2 \pm 1,2,4,1 \pm 1,4 \mathrm{ng} / \mathrm{ml}$ (moyennes \pm écarts types des moyennes)). II n'apparaît pas d'action additive ni synergique des 2 acides aminés. L'effet de l'arginine pourrait avoir lieu via une inhibition de la sécrétion de somatostatine, comme Alba-Roth et al (1989) l'ont observé chez l'homme.

L'effet peu marqué du tryptophane observé dans cet essai pourrait être dû à l'emploi d'une dose insuffisante. L'utilisation du tryptophane en solution plus concentrée est gênée par sa faible solubilité. Le tryptophane doit proba- blement exercer son action en stimulant le système sérotoninergique, et la sérotonine pourrait promouvoir la sécrétion de $\mathrm{GH}$ en accroissant celle de somatocrinine (Kakusca et Makara, 1983).

En conclusion, dans nos conditions expérimentales, l'injection intraveineuse de L-arginine provoque une augmentation du taux plasmatique de $\mathrm{GH}$, chez l'agneau ruminant. Le tryptophane, utilisé à dose relativement faible, ne procure qu'une petite augmentation. L'association de ces 2 acides aminés n'a pas d'effet additif ni synergique. Des études complémentaires sont nécessaires pour préciser l'influence de la dose, le mode d'action, ainsi que l'action sur la croissance de ces acides aminés.

Remerciements - Nous remercions la NIHDDK (Bethesda, MD, États-Unis) pour la fourniture gracieuse de $\mathrm{GH}$ ovine et d'antisérum anti-oGH.

Alba-Roth J, von Creytz C, Mehitretter G, von Weder $K$ (1989) Endocrinology 120 (suppl 1), 69-70

Davis SL (1972) Endocrinology 91, 549-555

Kakusca I, Makara GH (1983) Endocrinology $113,3 \uparrow 8-323$

Knopf RF, Conn JW, Fajans SS, Floyd JC, Guntsche EM, Rull JA (1965) J Clin Endocrinol Metab 25, 1140-1144 